\title{
BIBLIOGRAFÍA
}

\section{Contribución a la Bibliografía de Oliverio Girondo*}

\author{
SUMARIO
}

INTRODUCCION (por Nélida Salvador).

OBRAS DEL AUTOR (as. 1-74)

OBRAS COMPLETAS (as. 1)

LIBROS (as. 2-15)

COLABORACIONES EN PUBLICACIONES PERIODICAS (as. 16-35)

Prosa (as. 16-23)

Poesia (as. 24-35)

ANTOLOGIAS DE TEXTOS DEL AUTOR (as. 36)

TEXTOS DEL AUTOR EN DIVERSAS ANTOLOGIAS (as. 37-64)

PROLOGOS Y EDICIONES DE OBRAS DE OTROS AUTORES (as. 65-66)

TRADUCCIONES DEL AUTOR (as. 67-69)

TRADUCCIONES DE OBRAS DEL AUTOR (as. 70-73)

Francés (as. 70-71)

Inglés (as. 72)

Italiano (as. 73)

DISCOS (as. 74)

CRITICA Y BIOGRAFIA (as. 75-228)

LIBROS (as. 75-77)

*Por Nélida Salvador con la colaboración técnica de Elena Ardissone. 
CAPITULOS SOBRE EL AUTOR INCLUIDOS EN LIBROS (as. 78-103)

ARTICULOS EN PUBLICACIONES PERIODICAS (as. 104-218)

Trabajos firmados (as. 104-202)

Trabajos sin firmar (as. 203-218)

ARTICULOS Y MENCIONES SOBRE EL AUTOR EN HISTORIAS

DE LA LITERATURA, ENCICLOPEDIAS Y DICCIONARIOS (as. 219-228)

INDICE ALFABETICO DE AUTORES

ABREVIATURAS

\section{INTRODUCCION}

Oliverio Girondo, decisivo propulsor del movimiento "martinfierrista", es sin duda la figura más representativa del vanguardismo argentino. Su intensa trayectoria poética, caracterizada por una permanente actitud renovadora, en ningún momento desmintió la autenticidad de sus inconformistas ideales estéticos. Toda su labor creadora es un reiterado exponente de ese afán innovador que logró mantener sin claudicaciones a lo largo de casi cuatro décadas.

Sin embargo, tan acuciante desafío a la inercia y la mediocridad no ha recibido hasta hace pocos años - salvo aisladas excepciones-el reconocimiento crítico que sus méritos requieren. Después de 1960 la reedición de sus primeros libros y la inclusión de algunos poemas en numerosas muestras antológicas de la etapa vanguardista han contribuido a difundir la personalidad de Oliverio Girondo entre las jóvenes promociones. Por otra parte, la publicación de sus Obras completas (Buenos Aires, Losada, 1968), ha abierto nuevas perspectivas de indagación, intensificando el interés de la crítica por su quehacer poético a través de importantes estudios y artículos periodísticos.

Ese acrecentamiento de publicaciones, que viene acentuándose durante los últimos años, torna necesaria la ordenación sistemática de las mismas para facilitar su consulta a los investigadores. Con ese propósito hemos realizado esta Contribución a la bibliografía de Oliverio Girondo que, sin la pretensión de ser exhaustiva, procura actualizar y reordenar metodológicamente todos los materiales compilados hasta el momento. 


\section{OBRAS DEL AUTOR}

\section{OBRAS COMPLETAS}

1. Girondo, Oliverio. Obras completas. Buenos Aires, Losada, c. 1968. 487 p. retr., láms.

Contenido: Hacia el fuego central o la poesia de Oliverio Girondo, por Enrique Molina.- Cronología.-Veinte poemas para ser leidos en el tranvia. 1922: Carta abierta a "La Púa". Paisaje bretón. Café-concierto. Croquis en la arena. Nocturno. Rioo de Janeiro. Apunte callejero. Milonga. Venecia. Exvoto. Fiesta en Dákar. Croquis sevillano. Corso. Biarritz. Otro nocturno. Pedestre. Chioggia. Plaza. Lago Mayor. Sevillano. Verona.Calcomanias. 1925: Toledo. Calle de las sierpes. El tren expreso. Gibraltar. Tánger. Siesta. Juerga. Escorial. Alhambra. Semana Santa: Vísperas. Domingo de Ramos (mañana) (tarde). Miércoles Santo. Jueves Santo. Madrugada y tarde del Viernes Santo.Membretes. Espantapájaros (al alcance de todos). 1932: 1-24.Pintura moderna.- Interlunio.- Persuasión de los dias: 1. Vuelos sin orillas. Ejecutoria del miasma. ¡Azotadme! Aparición urbana. Arena. Testimonial. ¿Dónde? "Ruiseñor del lodo". Trì́ptico. Comunión plenaria. Atardecer. Es la baba.Nocturnos: 1-9.- 2. Rata-sirena-faústica. Invitación al vómito. Tótem. Derrumbe. Puedes juntar las manos. Cansancio. El. Visita. Hay que compadecerlos. - Embelecos: Nubífero anhelo. Nihilismo. Deserción. Dicotomía incruenta. Vórtice. Arborescencia. Restringido propósito. Salvamento. Predilección evanescente. Desmemoria. Escrúpulo. Pleamar. Fidelidad.- 3. Espera. Expiación. Rebelión de vocablos. A pleno llanto. Confidencia prosaica. Hazaña. Responso en blanco vivo. Dietética. Inagotable asombro. Lo que esperamos. Gratitud.-Campo nuestro.Poemas no reunidos en volumen: Figari pinta. Euforia. Nocturno. Encallado en las costas del Pacífico. Angelnorahcustodio.En la masmédula: La mezcla. Noche tótem. Al gravitar rotando. Canes más que finales. Aridandantemente. Islas sólo de sangre. Hay que buscarlo. Recién entonces. El uno nones. El pentotal a qué. El puro no. Rada anímica. Por vocación de dado. Mi lumia. Maspleonasmo. Alta noche. Trazumos. Tropos. Gristenia. Hasta morirla. Sopolsorbos. Las puertas. Yolleo. Posnotaciones. Plexilio. Ante el sabor inmóvil. Balaúa. Destino. Topatumba. Habría. Tantan yo. Porque me cree su perro. A mí. Manos. Mito. 
Ella. Cansancio.- Bibliografía de Oliverio Girondo, [por Horacio Jorge Becco? .- Indice de primeros versos.

\section{LIBROS}

2. ---, Calcomanias. Madrid, Calpe, 1925. No paginado "Las tapas y las viñetas, originales del autor, fueron iluminadas por A. Riosca."

3. ---, Campo nuestro. Buenos Aires, Sudamericana, c. 1946. 45 p.

4. --, En la masmédula. Buenos Aires, Losada, 1954. 56 p.

5. ---, ---. Buenos Aires, Losada, 1956. 80 p. (Poetas de España y América. Dir. Guillermo de Torre)

6. ---, En la masmédula; seguido de Yo tan yo, Destino, Topatumba, Cansancio, Mimito, Ella y otros poemas. Con una viñeta de Enrique Molina. 2. ed. Buenos Aires, Losada, 1963. 82 p. (Colección Poetas de ayer y de hoy)

7. ---, Espantapájaros (al alcance de todos). Buenos Aires, Proa, 1932. No paginado

Carátula de José Bonomi.

8. ---, Interlunio; relato en prosa. Ilus. de Lino Spilimbergo. Buenos Aires, Sur, 1937. No paginado

9. ---, Nuestra actitud ante el desastre. Buenos Aires, [s.e.], 1940. $47 \mathrm{p}$.

Comprende tres ensayos: el que da nombre al folleto; en Apéndice, dos artículos aparecidos en el diario La Nación el 25 de abril y el 21 de febrero de 1937, titulados Nuestra actitud ante Europa y El mal del siglo.

10. --, El periódico "Martin Fierro", 1924-1949; memoria de sus antiguos directores. Buenos Aires, F.A. Colombo, 1949. 58 p. "A pedido de los antiguos directores de Martin Fierro, el señor Córdova Iturburu leyó esta memoria en el acto organizado por la Comisión Directiva de la Sociedad Argentina de Escritores -con motivo de cumplirse veinticinco años de la aparición del periódico- el día 27 de octubre de 1949, en la Casa del Escritor..."

p. 59: "Esta memoria ha sido preparada y redactada por Oliverio Girondo, por encargo de sus ex compañeros en la Dirección del periódico Martín Fierro. Una vez adaptadas por el redactor las observaciones y sugestiones que ellos efectuaron, fue 
plenamente aprobada y firmada por los cuatro. Evar Méndez, Oliverio Girondo, Alberto Prebisch, Eduardo J. Bullrich.

11. ---, Persuasión de los dias. Buenos Aires, Losada, c. 1942. 185 p. (Poetas de España y América. Dir. Amado Alonso y Guillermo de Torre)

12. ---, Topatumba. Buenos Aires, 1958. No paginado

Dib. de Enrique Molina en las prensas de Miguel Binolo. Carpeta realizada por Francisco A. Colombo. Véase as. 6

13. ---, Veinte poemas para ser leidos en el tranvia. Illus. del autor. Argenteuil (Francia), Impr. Coulouman H. Barthélemy, 1922. No paginado

14. ---, ---.. Illus. del autor. 2. ed. Buenos Aires, Editorial Martín Fierro, 1925. No paginado

"Edición tranviäria a veinte centavos. Se agrega como prólogo una Carta abierta a 'La Pua' (Parîs, dic. de 1922) y unas líneas a Evar Méndez."

15. -.., ---. Calcomanias. Espantapájaros. Buenos Aires, Centro Editor de América Latina, 1966. 110 p. (Serie del encuentro, 5)

Contenido: Veinte poemas para ser leidos en el tranvia: Carta abierta a "La Púa". Paisaje bretón. Café-concierto. Croquis en la arena. Nocturno. Rỉo de Janeiro. Apunte callejero. Milonga. Venecia. Exvoto. Fiesta en Dákar. Croquis sevillano. Corso. Biarritz. Otro nocturno. Pedestre. Chioggia. Plaza. Lago Mayor. Sevillano. Verona.-Calcomanias: Toledo, Calle de las sierpes. El tren expreso. Gibraltar. Tánger. Siesta. Juerga. Escorial. Alhambra. Semana Santa: Vìsperas. Domingo de Ramos. Miércoles Santo. Jueves Santo. Madrugada y tarde del Viernes Santo.Espantapájaros: 1 a 24 .

Véase as. 1

\section{COLABORACIONES EN PUBLICACIONES PERIODICAS}

Prosa (orden cronológico)

16. ---, Membretes. (En: Martǐn Fierro, Buenos Aires, 2. ép., a. 1, no 1 , feb. 1924; no 4, 15 mayo, 1924; n० 8-9, ag.-set. 1924. a. 2 , no 18,26 jun. 1925; n० 23, 25 set. 1925; a. 3, no 32, 4 ag. $1926 ; n^{\circ} 34,5$ oct. 1926 )

Véase as. 1 
17. --, Carta abierta a "La Púa". (En: Martîn Fierro, Buenos Aires, 2. ép., a. 1, no 2, 20 marzo, 1924)

Se incluyen también los poemas: Paisaje bretón, Croquis en la arena, Nocturno, Rio de Janeiro, Milonga, Exvoto, Fiesta en Dákar y Otro nocturno.

Véase as. 1,13 y 15

18. --, [Respuesta] . (En: Martín Fierro, Buenos Aires, 2. ép., a. 1, no 5-6, 15 mayo-15 jun. 1924)

"Contestaciones a la encuesta de Martin Fierro: $1^{\circ}{ }_{¿}$ Cree Vd. en la existencia de una sensibilidad, de una realidad argentina? .$2^{\circ}$ En caso afirmativo; ¿cuáles son sus caracterîsticas?".

19. ---, Una entrevista con Oliverio Girondo. (En: Proa, Buenos Aires, a. 1, no 4, nov. 1924, pp. 62-63)

En Variedades, Lima.

20. ---, Radiograma a Ramón. (En: Martīn Fierro, Buenos Aires, 2. ép., a 2, no 19, 18 jul. 1925)

Homenaje a Ramón Gómez de la Serna.

21. --, Monumento a Hernández. (En: Martĭn Fierro, Buenos Aires, 2. ép., a. 2, no 22, 10 set., 1925)

Carta de Oliverio Girondo a Evar Méndez, para proponer que Martin Fierro auspicie la idea de erigir un monumento a José Hernández. Respuesta de la Dirección.

22. ---, Cuidado con la arquitectura. (En: Martín Fierro, Buenos Aires, 2. ép., a. 2, no 24, 17 oct. 1925, ilus.)

23. --, [Autobiografía breve] . (En: Martǐn Fierro, Buenos Aires, 2. ép., a. 4, no 39, 28 marzo, 1927)

"Algunas páginas de la Exposición de la actual poesia argentina, por Pedro Juan Vignale y César Tiempo."

Poesia (orden cronológico)

24. --, Nocturno, Corso y Chioggia. (En: Mundial, Lima, n० 221, 8 ag. 1924) Véase as. 13 y 15

25. --, Croquis en la arena, Nocturno y Otro nocturno. (En: El Universal, México, 5 oct. 1924)

Véase as. 1,13 y 15

26. ---, Escorial y Juerga. (En: Revista de Occidente, Madrid, t. 7, no 19 , en.-marzo 1925 , pp. 28-32) 
Poesĩas que luego formaron parte del libro Calcomanias. Véase as. 1,2 y 15

27. ---, Nuevos poemas de "Calcomanias". (En: Martǐn Fierro, Buenos Aires, 2, ép., a. 2, no 16, 5 mayo, 1925)

Contiene: Escorial y Juerga.

Véase as. 1,2 y 15

28. ---, Insomnio. (En: Proa, Buenos Aires, a. 2, no 11, jun. 1925, p. 24)

29. --, Deux poèmes. (En: La Revue argentine, Paris, a. 1, no 1, juin 1934, pp. 21-22)

Tr. Pièrre-Charles Roncal.

Contenido: Nocturne. Autre nocturne.

Véase as. 1,13 y 15

30. --, Quatre croquis d'album. (En: La Revue argentine, Paris, a. 1, no 6, fév. 1935, p. 7-9)

Tr. Pièrre-Charles Roncal.

Contenido: Río de Janeiro. Séville. Vérone. Venise.

Véase as. 1,13 y 15

31. --, Epuvantails. (En: La Revue argentine, Paris, no 12, oct.-nov. 1935)

Tr. Manoel Gahisto.

Véase as. 1,7 y 15

32. ---, Interlune. (En: La Revue argentine, Paris, a. 5, n० 26, juin 1938, pp. 9-19)

Tr. Manoel Gahisto.

Véase as. 1 y 8

33. ---, Nocturno 2 y El pentotal a qué. (En: Poesia Buenos Aires, Buenos Aires, no 21 verano 1956, pp. 12-13)

Véase as. 1 y 5

34. ---, Cansancio. (En: El Angel del altillo, Buenos Aires, a. 1, no 1, mayo 1967, p. 14)

Véase as. 1 y 6

35. ---, Dicotomia incruenta (de "Persuasión de los días"). Grabado de Alberto J. Borzone. (En: Artes y letras argentinas, Buenos Aires, Fondo Nacional de las Artes, a. 8, no 3020 poetas, 20 grabadores, mayo 1967 , pp. 20-21)

Véase as. 1 y 11 


\section{ANTOLOGIAS DE TEXTOS DEL AUTOR}

36. ---, Oliverio Girondo, por Aldo Pellegrini. Buenos Aires, Ediciones culturales argentinas, Ministerio de Educación y Justicia, Dirección General de Cultura, c. 1964.132 p. (Colección Antologías)

Contenido: Mi visión personal de Oliverio Girondo.- Breve biografía.-La poesia de Girondo.-Veinte poemas para ser leidos en el tranvia. -Calcomanias.- Espantapájaros.-Persuasión de los días.- Campo nuestro.- En la masmédula.-. Inéditos: Porque me cree su perro. Cansancio.

Véase as. 1 y 15

\section{TEXTOS DEL AUTOR EN DIVERSAS ANTOLOGIAS}

37. ---, Calle de las sierpes y Al gravitar rotando. (En: Isaacson, José - Urquía, Carlos Enrique. 40 años de poesía argentina 1920-1960. Buenos Aires, Aldaba, 1962-64. v. 1, pp. 98-101)

Precedido de una breve biografía.

38. ---, Campo nuestro (fragmentos). (En: Bartholomew, Roy. Cien poesias rioplatenses, 1800-1950. Antología, ordenación, pról., notas sobre la poesía en el Río de la Plata y bio-bibliográficas de los poetas. Buenos Aires, Raigal, c. 1954. pp. 277-279)

39. ---, Cansancio y Testimonial. (En: La poesia del siglo veinte en América y España. Buenos Aires, Ediciones de la revista "Caballo de fuego", 1952. pp. 280-283)

40. ---, Croquis en la arena, Milonga y Calle de las sierpes. (En: Baciu, Stefan. Antologia de la poesia surrealista latinoamericana. México, J. Mortiz, c 1974. pp. 141-144)

Oliverio Girondo en el tranvia, por Stefan Baciu, pp. 53-56.

41. --, Croquis en la arena, Paisaje bretón, Nocturno, Otro nocturno, y Tánger. (En: Prieto, Adolfo. Antología de Boedo y Florida. Pról. y selec. Córdoba, Universidad Nacional de Córdoba, 1964. Colección El Pensamiento Argentino. pp. 119-125)

42. --, Escorial. (En: Córdova Iturburu, Cayetano. La revolución martinfierrista. Buenos Aires, Ediciones culturales argentinas, Ministerio de Educación y Justicia, Dirección General de Cultura, 1962. Biblioteca del Sesquicentenario. Colección Movimientos literarios. pp. 56-58) 
43. --, Espantapájaros, no 2, 4 y 6. (En: Sorrentino, Fernando. Cuentos argentinos con humor; siglo XX. Buenos Aires, Plus ultra, c. 1977. pp. 108-112)

44. ---, [Espantapájaros] no 11. (En: Sorrentino, Fernando. selec. y notas bio-bibliográficas. 35 cuentos breves argentinos; siglo $X X$. Buenos Aires, Plus ultra, c. 1977. p. 92-94)

45. ---, Exvoto, a las chicas de Flores y Apunte callejero. (En: Salas, Horacio. La poesia de Buenos Aires; ensayo y antologia. Buenos Aires, Pleamar, c. 1968. Colección Mar abierto. pp. 177-180)

46. ---, Insomnio y [Más que tierra eres cielo]. (En: Ara, Guillermo. Suma de poesía argentina (1538-1968); critica y antologia. Buenos Aires, Guadalupe, c. 1970. v. 2, p. 74)

Referencias críticas sobre el autor en el v. 1.

Véase as. 28 y 54

47. --, Islas sólo de sangre. (En: Abril, Xavier. Breve antología de la poesia moderna hispanoamericana. Bahïa Blanca, Universidad Nacional del Sur, Extensión Cultural, c. 1959. Serie: El Viento. pp. 12-16)

Referencia crǐtica sobre el autor, por Xavier Abril.

48. ---, Juerga y Calcomanias. (En: González Climent, Anselmo. Antologia de poesia flamenca. Madrid, Escelier, 1961. Colección $21, \mathrm{n}^{\circ} 31$, p. 186 )

49. --, La mezcla, Noche tótem, Hay que buscarlo, Trazumos, Tropos, Ante el sabor inmóvil, Destino, A mi. (En: Rosales, César, selec. Antologia de la poesia argentina contemporánea. Pról. de Guillermo de Torre. Buenos Aires, Ministerio de Relaciones Exteriores y Culto, Dirección General de Relaciones Culturales, 1964. pp. 47-55)

50. --, Milonga, Exvoto, Juerga, y Mi lumia. (En: Martini Real, Juan Carlos. Los mejores poemas de la poesia argentina. Buenos Aires, Corregidor, c. 1974. pp. 61-65)

Precedido de un comentario.

51. --, No se me importa un pito. . . (En: Fernández Moreno. La realidad y los papeles; panorama de la poesia argentina contemporánea. Madrid, Aguilar, 1967. c. 1961. (Biblioteca Cultura e historia. p. 513)

52. --, Noctumo, Calle de las sierpes y fragmentos de $E s^{-}$ pantapájaros: 3, 18 y 9. (En: González Carbalho, José. Indice de la poesia argentina contemporánea. Santiago de Chile, Ercilla, 1937. pp. 221-225) 
53. ---, Nocturno, Capitulo dos, Capitulo dieciseis y Capitulo veintiuno. (En: Murray, Luis Alberto. Selec., pról. y notas biográficas. Humorismo argentino. Buenos Aires, Ediciones culturales argentinas, Ministerio de Educación y Justicia, Dirección General de Cultura, 1961. Biblioteca del Sesquicentenario. Colección Antologías. pp. 91-97)

54. ---, Nocturno, Otro nocturno, Escorial, Juerga e Insomnio. (En: Vignale, Pedro Juan - Tiempo, César. Exposición de la actual poesia argentina (1922-1927). Buenos Aires, Minerva, 1927. pp. 15-21)

Precedidos de una caricatura y breve autobiografïa. Véase as. 28 y 46

55. ---, Nocturno y Otro nocturno. (En: Borges, Jorge Luis Ocampo, Silvina - Bioy Casares, Adolfo. Antologia poética argentina. Buenos Aires, Sudamericana, c. 1941.pp. 145-146)

56. ---, Otro nocturno, Lago Mayor, Calle de las sierpes, Aparición urbana, Es la baba, Lo que esperamos, Campo nuestro (fragmentos), Tropos, y A mi. (En: Jiménez, José Olivio. Selec., pról. y notas. Antologia de la poesia hispanoamericana contemporánea: 1914-1970. 2. ed. Madrid, Alianza editorial, 1973. El Libro de bolsillo, 289. pp. 90-102)

57. ---, Otro nocturno, Siesta, Alhambra (fragmentos), Insomnio y Espantapájaros. (En: Ara, Guillermo. Selec. Los poetas de Florida. Buenos Aires, Centro Editor de América Latina, c. 1968. Capítulo; Biblioteca argentina fundamental, 40. pp. 21-23)

58. ---, Paisaje bretón, Calle de las sierpes, Nocturno 4, Campo nuestro, Recién entonces, y $A$ mi. (En: Fernández Moreno, César - Becco, Jorge Horacio. Comp. Antologia lineal de la poesia argentina. Madrid, Gredos, c. 1968. Biblioteca románica hispánica, 26. VI. Antología hispánica. pp. 275-284)

Precedido de un comentario.

59. ---, Responso en blanco vivo. (En: Antologia de la poesia moderna hispanoamericana. Montevideo, 1956. Cuadernos Julio Herrera y Reissig, no 46. pp. 10-11)

60. ---, Rio de Janeiro. (En: González Lanuza, Eduardo. Los martinfierristas. Buenos Aires, Ediciones culturales argentinas, Ministerio de Educación y Justicia, Dirección General de Cultura, 1961. Biblioteca del Sesquicentenario. Colección Movimientos literarios. pp. 50-51) 
61. ---, [Se miran, se presienten, se desean], [Abandoné las carambolas por el calambur, los madrigales por los mamboretás...] y Restringido propósito. (En: Poesia lírica del siglo XX: España e Hispanoamérica. Buenos Aires, Centro Editor de América Latina, c. 1972. Biblioteca fundamental del hombre moderno, 56. pp. 25-27)

62. ---, Toledo. (En: Onǐs, Federico de. Antologia de la poesía española e hispanoamericana (1882-1932). New York, Las Américas Publishing Co., 1961. pp. 993-995)

63. ---, Toledo, Calle de las sierpes y Tánger. (En: Noé, Julio. Antologia de la poesia argentina moderna (1900-1925). Con notas biográficas y bibliográficas. Buenos Aires, Nosotros, 1926. pp. 503-511)

64. ---, Vuelo sin orillas, Salvamento y Espera. (En: Caillet-Bois, Julio -Rossi de Fiori, Iride. 25 poetas argentinos 1920-1945. Buenos Aires, Eudeba, c. 1964. Serie del Siglo y medio, 58. pp. 45-49)

\section{PROLOGOS Y EDICIONES DE OBRAS DE OTROS AUTORES}

65. Pintura moderna. Colección de Rafael A. Crespo. Pról. de Oliverio Girondo. Buenos Aires, Impr. Colombo, 1936. 325 p. ilus.

Exposición en el Museo Nacional de Bellas Artes, Buenos Aires, agosto 14 al 6 de setiembre de 1936.

66. Supervielle, Jules. Choix de poèmes. Buenos Aires, Impr. F.A. Colombo, 1944. 285 p.

Libro de homenaje a Jules Supervielle.

Dirigieron esta edición Eduardo J. Bullrich y Oliverio Girondo.

\section{TRADUCCIONES DEL AUTOR}

67. Rimbaud, Jean Arthur. Una temporada en el Infierno (fragmento). Tr. de Oliverio Girondo y Enrique Molina. (En: Galtier, Lysandro Z.D. La traducción literaria; ensayo. Con una antologìa del poema traducido. Buenos Aires, Ediciones culturales argentinas, Ministerio de Educación y Justicia, Subsecretarìa de Cultura, c. 1965. v. 2, pp. 189-190)

68. ---, ---. Versión castellana de Oliverio Girondo y Enrique Molina. Buenos Aires, Compañia General Fabril Editora, 1959. 
77 p. retr. (Los Poetas)

69. ---, ---. Tr. Oliverio Girondo y Enrique Molina. Buenos Aires, Compañia General Fabril Editora, 1971. 77 p. (Los Poetas)

Tít. orig.: Une saison en Enfer.

TRADUCCIONES DE OBRAS DEL AUTOR

Francés

70. Girondo, Oliverio. Epouvantails. Tr. Manoel Gahisto. (En: Galtier, Lysandro Z.D. La traducción literaria; ensayo. Con una antología del poema traducido. Buenos Aires, Ediciones culturales argentinas, Ministerio de Educación y Justicia, Subsecretaría de Cultura, c. 1965. v. 3: Autores argentinos traducidos a otros idiomas, pp. 82-86)

Véase as. 31

71. ---, Nocturne. Tr. Pièrre-Charles Roncal. (En: Galtier, Lysandro Z.D. La traducción literaria; ensayo. Con una antología del poema traducido. Buenos Aires, Ediciones culturales argentinas, Ministerio de Educación y Justicia, Subsecretaría de Cultura, c. 1965. v. 3: Autores argentinos traducidos a otros idiomas, p. 77)

Véase as. 29

\section{Inglés}

72. ---, Las Sierpes Street. Tr. Milton Ben Davis. (En: Galtier, Lysandro Z.D. La traducción literaria; ensayo. Con una antologìa del poema traducido. Buenos Aires, Ediciones culturales argentinas, Ministerio de Educación y Justicia, Subsecretaria de Cultura, c. 1965. v. 3: Autores argentinos traducidos a otros idiomas, pp. 80-81)

De Anthology of Contemporary Latin-American Poetry, ed. by Dudley Fitts. Norfolk, Conn., 1942.

\section{Italiano}

73. ---, Via delle serpi. Tr. Folco Testena. (En: Galtier, Lysandro Z.D. La traducción literaria; ensayo. Con una antología del 
poema traducido. Buenos Aires, Ediciones culturales argentinas, Ministerio de Educación y Justicia, Subsecretaría de Cultura, c. 1965. v. 3: Autores argentinos traducidos a otros idiomas, pp. 77-80)

Versión bilingüe.

De Antología della poesia argentina moderma, por Folco Testena. Milano, Alpes, 1927.

\section{DISCOS}

74. --, En la masmédula y Cansancio. Tapa de Luis Seoane. Buenos Aires. 2 f., 30 cm., 33 r.p.m. (Colección Palabra en el tiempo. Dir. Arturo Cuadrado y Carlos Mazzanti)

\section{CRITICA Y BIOGRAFIA}

\section{LIBROS}

75. Corro, Gaspar Pío del. Oliverio Girondo; los limites del signo. Buenos Aires, Fernando García Cambeiro, c. 1976. 205 p. (Colección Estudios latinoamericanos. Dir. Graciela Maturo)

76. De Nóbile, Beatriz. El acto experimental; Oliverio Girondo y las tensiones del lenguaje. Buenos Aires, Losada, c. 1972. 174 p. (Biblioteca de estudios literarios)

Bibliografía: pp. 161-172.

77. Scrimaglio, Marta. Oliverio Girondo. Santa Fe, Universidad Nacional del Litoral, Facultad de Filosofía y Letras, 1964. 61 p. (Cuadernos del Instituto de Letras)

\section{CAPITULOS SOBRE EL AUTOR INCLUIDOS EN LIBROS}

78. Ara, Guillermo. Los argentinos y la literatura nacional; estudios para una teoria de nuestra expresión. Buenos Aires, Huemul, 1966. 155 p. (Estudios de literatura, 1)

Sobre Oliverio Girondo, pp. 51-52.

79. Carilla, Emilio. El vanguardismo en la Argentina (sobre un momento literario y una revista). Resistencia, Universidad Nacional del Nordeste, 1961. pp. 51-82. 
Separata de la revista Nordeste, Resistencia, Universidad Nacional del Nordeste, no 1, 1961, pp. 51-82.

De sus Estudios sobre literatura argentina; siglo XX. Tucumán, Universidad Nacional de Tucumán, Facultad de Filosofía y Letras, 1961. 161 p. (Cuadernos de Humanitas, no 6)

80. Córdova Iturburu, Cayetano. La revolución martinfierrista. Buenos Aires, Ediciones culturales argentinas, Ministerio de Educación y Justicia, Dirección General de Cultura, 1962. 194 p. (Biblioteca del Sesquicentenario. Colección Movimientos literarios)

81. Dỉez Canedo, Enrique. Girondo. (En su: Letras de América. México, El Colegio de México, 1944.pp. 364-368)

82. Fernández Moreno, César. La realidad y los papeles; panorama y muestra de la poesia argentina contemporánea. Madrid, Aguilar, 1967, c. 1961.633 p. (Biblioteca Cultura e historia)

Sobre Oliverio Girondo p. 149, especialmente.

83. Garcỉa, Germán. La novela argentina; un itinerario. Buenos Aires, Sudamericana, c. 1952. 317 p.

Sobre Oliverio Girondo, pp. 160 y 290.

84. Ghiano, Juan Carlos. Oliverio Girondo. (En su: Poesía argentina del siglo XX. México-Buenos Aires, Fondo de Cultura Económica, 1957. Colección Tierra firme, 65. pp. 112-117)

85. Gómez de la Serna, Ramón. Oliverio Girondo. (En sus: Retratos completos. Madrid, Aguilar, 1961.pp. 323-340)

86. ---, ---. (En sus: Retratos contemporáneos. Buenos Aires, Sudamericana, 1941. pp. 83-101)

87. ---, ---. (En sus: Retratos contemporáneos escogidos. Buenos Aires, Sudamericana, 1968. Colección Piragua. pp. 189-212)

88. González Lanuza, Eduardo. Los martinfierristas. Buenos Aires, Ediciones culturales argentinas, Ministerio de Educación y Justicia, Dirección General de Cultura, 1961. 142 p. (Biblioteca del Sesquicentenario. Colección Movimientos literarios)

89. Ibarra, Néstor. Los humoristas: Girondo. (En su: La nueva poesia argentina; ensayo critico sobre el ultraismo, 1921-1929. Buenos Aires, Impr. Vda. de Molinari, 1930. pp. 92-94)

90. Lange, Norah. A Oliverio Girondo y A Oliverio Girondo con motivo de Interlunio. (En sus: Discursos. Buenos Aires, Ediciones C.A.Y.D.E., 1942. pp. 31-36, 63-68 y 89-95)

91. Ledesma, Roberto. Una geografia argentina vista por poetas. Buenos Aires, Ediciones culturales argentinas, Ministerio de E- 
ducación y Justicia, Dirección General de Cultura, 1964. 133 p. (Colección Antologías)

92. Mazzei, Angel. El modernismo en la Argentina. La poesia de Buenos Aires. Buenos Aires, Ciordia, 1962. 217 p.

93. Pinto, Juan. Breviario de literatura argentina contemporánea. Buenos Aires, La Mandrágora, 1958. 300 p.

Sobre Oliverio Girondo, pp. 162-163 y en Apéndice: Manifiesto de Martin Fierro, pp. 280-282.

94. ---, Literatura argentina del siglo XX. Buenos Aires, Ediciones argentinas, 1943.

Sobre Oliverio Girondo, pp. 166-167.

95. --, Oliverio Girondo. (En su: Panorama de la literatura argentina. Buenos Aires, Mundi, 1941.pp. 175-176)

96. Prieto, Adolfo. El periódico “Martin Fierro". Buenos Aires, Galerna, 1968. 214 p. (Colección Las Revistas, n० 1)

Manifiesto de Martin Fierro, pp. 13-15. - Encuesta, respuesta de Oliverio Girondo, pp. 88-89.- Radiograma a Ramón Gómez de la Serna, pp. 128-129.- Membretes, pp. 193-195.

Véase as. 10,18 y 20

97. Salvador, Nélida. Revistas argentinas de vanguardia (1920-1930). Buenos Aires, Universidad de Buenos Aires, Facultad de Filosofía y Letras, Instituto de Literatura Argentina "Ricardo Rojas", 1962. 105 p.

98. Sofovich, Luisa. Ramón Gómez de la Serna. Buenos Aires, Ediciones culturales argentinas, Ministerio de Educación y Justicia, Dirección General de Cultura, 1962. 213 p.

Se incluye el retrato sobre Oliverio Girondo, pp. 61-80.

Véase as. 86

99. Sola, Graciela de. Oliverio Girondo. (En su: Proyecciones del surrealismo en la literatura argentina. Buenos Aires, Ediciones culturales argentinas, Secretaria de Estado de Cultura y Educación, c. 1967. Colección Movimientos literarios. pp. 84-92)

100. Sucre, Guillermo. Lenguaje y conciencia critica. Adiciones adhesiones. (En su: La máscara, la transparencia; ensayos sobre poesia hispanoamericana. Caracas, Monte Avila, 1975. pp. 273-285)

101. Torre, Guillermo de. Ultraismo en América hispánica. Argentina. Oliverio Girondo. (En su: Historia de las literaturas de vanguardia. Madrid, Ediciones Guadarrama, c. 1965. p. 584) 
102. Victoria, Marcos. El humorismo en la literatura argentina actual. (En sus: Variaciones sobre lo sentimental. Buenos Aires, Sudamericana, 1944. pp. 231-257)

103. --, Sobre Oliverio Girondo. (En su: El viajero y los paisajes. Primera serie. Buenos Aires, M. Gleizer, 1934. pp. 149-154)

\section{ARTICULOS EN PUBLICACIONES PERIODICAS}

Trabajos firmados

104. A.G. Motivos cotidianos. Un poeta argentino: Girondo. (En: Crónica, Lima, 5 ag. 1924)

105. Abril, Xavier. La evolución de la poesia moderna. (En: Cuadernos del Congreso por la libertad de la cultura, Parǐs, no 19, jul.-ag. 1956, pp. 131-136)

106. Aguilera, Ricardo F. Poesia argentina: Girondo. (En: Crónica veracruzana, México, no 3, oct. 1947, pp. 17-19)

107. Aguirre, Raúl Gustavo. Oliverio Girondo. (En: Poesía Buenos Aires, Buenos Aires, no 21, verano 1956, p. 14)

108. Aita, Antonio. Notas al margen de la poesia argentina. (En: Nosotros, Buenos Aires, a. 23, t. 63, no 236-237, en.-feb. 1929, pp. 18-29)

109. Alberti, Rafael. Poemas escénicos. . (Boletín de la Sociedad Argentina de Escritores, SADE, Buenos Aires, 1961-1963, p. 126)

Forma parte del homenaje a Oliverio Girondo realizado en la SADE, el 30 de noviembre de 1962, celebrando sus Veinte poemas para ser leidos en el tranvia.

110. Alomar, Gabriel. A propósito de "Veinte poemas para ser leidos en el tranvia", por Oliverio Girondo. (En: La Nación, Buenos Aires, 23 dic. 1923, supl., p. 9)

De El Imparcial, Madrid, 2 set. 1923.

111. Aznar, Luis. Oliverio Girondo: "Calcomanias". (En: Valoraciones, La Plata, a. 1, no 7, set. 1925, pp. 73-78)

112. Barletta, Leónidas. A la muerte de Oliverio Girondo. (En: Propósitos, Buenos Aires, no 173, 2 feb. 1967)

113. Barrantes Castro, Pedro. El poeta Oliverio Girondo. (En: Mundial, Lima, no 221, 8 ag. 1924)

114. Bayley, Edgar. Homenaje a Oliverio Girondo; poesia. (En: Macedonio, Buenos Aires, a. 1, no 3, invierno 1969; pp. 64-65)

115. Becco, Horacio Jorge. El "vanguardismo" en la Argentina 
(1920-1930). (En: Cuadernos del idioma, Buenos Aires, a. 1, no 4, abr. 1966, pp. 127-152)

116. Bernárdez, Francisco Luis. Poesia argentina. (En: Mundo hispánico, Madrid, no 148, jul. 1960, pp. 88-91)

117. Bolaños, Federico. Oliverio Girondo. (En: Flechas, Lima, 29 nov. 1924)

118. Borges, Jorge Luis. Oliverio Girondo: "Calcomanias". (En: Martǐn Fierro, Buenos Aires, 2. ép., a. 2, no 18, 26 jun. 1925)

Con el mismo tít. apareció en La Epoca, Buenos Aires, 20 jun. 1925.

En El tamaño de mi esperanza. Buenos Aires, Proa, 1926, recoge este trabajo en las pp. 92-95).

119. Capdevila, Arturo. "Espantapájaros; al alcance de todos", por Oliverio Girondo. (En: Nosotros, Buenos Aires, a. 26, v. 77, no 281 , oct. 1932, p. 108)

120. Cárdenas de Monner Sans, Marïa Inés. "Martin Fierro", ¿revista, grupo o generación? (En: Universidad, Santa Fe, Universidad Nacional del Litoral, no 42, oct.-dic. 1959, pp. 5-23)

121. Casasbellas, Ramiro de. Oliverio, el principe de los poetas. (En: Primera plana, Buenos Aires, a. 5, no 214, 31 en.- 6 feb. 1967, pp. 60-61)

122. Cassou, Jean. Oliverio Girondo. (En: La Révue de l'Amérique Latine, Paris, set. 1923)

Sobre Veinte poemas para ser leidos en el tranvia.

123. Cometta Manzoni, Aìda. Figuras de "Martin Fierro": Oliverio Girondo. (En: El Universal, Buenos Aires, 12 nov. 1950, supl. literario)

124. Córdova Iturburu, Cayetano. Encomio de Oliverio Girondo. (En: Boletín de la Sociedad Argentina de Escritores, SADE, Buenos Aires, 1961-1963, pp. 127-129)

Forma parte del homenaje a Oliverio Girondo realizado en la SADE, el 30 de noviembre de 1962, celebrando sus Veinte poemas para ser leidos en el tranvia.

125.--, El heroico humorismo de "Martin Fierro". (En: El Sol, Madrid, 29 oct. 1939)

126. Delio, H.B. El "Espantapájaros", de Girondo. (En: Nosotros, Buenos Aires, a. 26, v. 77, no 281, oct. 1932, pp. 123-124)

127. Diego, Celia de. Oliverio Girondo. (En: La Prensa, Buenos Aires, 11 jul. 1965) 
Sobre el libro Oliverio Girondo, con introd. de Aldo Pellegrini. 128. Diez Canedo, Enrique. "Veinte poemas para ser leidos en el tranvia", por Oliverio Girondo. (En: España, Madrid, no 386, 8 set. 1923)

Publicado también en Nosotros, Buenos Aires, a. 17, v. 45, no 174, nov. 1923, pp. 386-388, y en La Nación, Buenos Aires, 23 dic. 1923 , p. 9.

129. Donoso, Armando. Un poeta: Oliverio Girondo (En: Zig-Zag, Santiago de Chile, 19 jul. 1924)

130. Erro, Carlos Alberto. A los cuarenta años de un celebrado libro de Oliverio Girondo. (En: Boletìn de la Sociedad Argentina de Escritores, Buenos Aires, 1961-1963, pp. 125-126)

Forma parte del homenaje a Oliverio Girondo realizado en la SADE, el 30 de noviembre de 1962, celebrando sus Veinte poemas para ser leidos en el tranvia.

131. F.B. Glosas bibliográficas: "Veinte poemas para ser leidos en el tranvia". (En: Flechas, Lima 29 nov. 1924)

132. Fernández Castro, Alberto. Oliverio Girondo. (En: La Noticia, Bogotá, 29 set. 1925)

133. Fernández Moreno, César. Girondo entre dos calles de Buenos Aires. (En: Casa de las Américas, La Habana, no 42, 5 jun. 1967, pp. 143-147)

134. Ferreiro, Alfredo Mario. Una nota sobre la vida y la obra del escritor Oliverio Girondo. (En: La Razón, Montevideo, 7 dic. 1942)

135. G.C. Oliverio Girondo, "Calcomanias", (En: El Sol, Madrid, 10 abr. 1925)

136. Ganduglia, Santiago. "Persuasión de los dias", por Oliverio Girondo. (En: Noticias gráficas, Buenos Aires, dic. 1942)

137. Giménez Caballero, Ernesto. España en América y América en España: "Cinelandia"; "Calcomanias". (En: El Sol, Madrid, 20 abr. 1925)

Sobre las obras de Ramón Gómez de la Serna y Oliverio Girondo, respectivamente.

138. Gimferrer, Pedro. En la muerte de un gran poeta, Oliverio Girondo. (En: Destino, Madrid, feb. 1967)

139. Giusti, Roberto F. "Interlunio", por Oliverio Girondo. (En: Nosotros, Buenos Aires, 2. ép., a. 3, v. 6, no 23, feb. 1938, pp. 226-227) 
140. Gómez Carrillo, Enrique. Un premio literario que se convierte en un escándalo parisiense. (En: La Razón, Buenos Aires, 12 ag. 1923)

141. Gómez de la Serna, Ramón. Oliverio Girondo; silueta total a propósito de su nuevo libro "Interlunio". (En: Sur, Buenos Aires, no 40, en. 1938, pp. 59-71)

142. ---, "Veinte poemas para ser leidos en el tranvia", por Oliverio Girondo. (En: Nosotros, Buenos Aires, a. 18, v. 46, no 176, en. 1924, pp. 137-138)

Tomado de El Sol, Madrid, 4 mayo 1923.

143. González Contreras, Gilberto. Oliverio Girondo: "Persuasión de los dias". (En: Revista Iberoamericana, México, v. 10, 1946, pp. 356-359)

144. González Lanuza, Eduardo. Oliverio Girondo: "Persuasión de los dias". (En: Sur, Buenos Aires, no 97, oct. 1942, pp. 102-107)

145. Goyné, André. Girondo; poesia. (En: Macedonio, Buenos Aires, a. 1, no 3 , invierno, 1969 , p. 79)

146. J.H.B. Oliverio Girondo a través de una antologia. (En: La Nación, Buenos Aires, 23 en. 1966)

147. Jarnés, Benjamín. Oliverio Girondo: "Calcomanias". (En: Revista de Occidente, Madrid, t. 8, mayo 1925, pp. 255-257)

148. Latorre, Carlos. "En la masmédula" y Oliverio Girondo. (En: Cultura universitaria, Caracas, Universidad Central de Venezuela, Dirección de Cultura, no 68-69, jul.-dic. 1959, pp. 140-144)

149. ---, --.. (En: Argentores, Buenos Aires, a. 31, no 124, jul.-dic. 1966, pp. 13-15)

150. Loprete, Carlos, Alberto. "En la masmédula", por Oliverio Girondo. (En: Ficción, Buenos Aires, no 10, nov.-dic. 1957, pp. 163-164)

151. M.C. "Calcomanias", de Oliverio Girondo. (En: La Vanguardia, Buenos Aires, 26 jul. 1925)

152. Madariaga, Francisco. Viaje al Paraguay con Oliverio. (En: Macedonio, Buenos Aires, a. 1, no 3, invierno, 1969, pp. 66-67)

153. Marechal, Leopoldo. "Interlunio", por O. Girondo. (En: Sur, Buenos Aires, no 48, set. 1938, pp. 51-53)

154. Martǐnez Cuitiño, Vicente. Oliverio Girondo $y$ sus veinte poemas. (En: Vida nuestra, Buenos Aires, a. 7, no 3, set. 1923) 
Reproducido en Critica, Buenos Aires, 23 dic. 1923 y en Renovación, Buenos Aires, abr. 1924.

155. Maseras, Alfonso. Oliverio Girondo. (En: La Veu de Catalunya, Barcelona, 8 jun. 1923)

156. Méndez, Evar. Doce poetas nuevos. (En: Síntesis, Buenos Aires, a. $1, n^{\circ} 4,1927$, pp. 15-33)

157. --, La generación de poetas del periódico "Martín Fierro". (En: Contrapunto, Buenos Aires, a. 1, no 5, ag. 1945, pp. 8-9 y 13-14)

158. ---, La joven literatura argentina. (En: El Orden, Tucumán, 31 dic. 1924)

159. Miomandre, Francis de. Oliverio Girondo. (En: La Nación, Buenos Aires, 20 en. 1924)

160. Mister Pin. La novisima literatura argentina: "Calcomanias", de Oliverio Girondo. (En: La Plata, Montevideo, 5 jun. 1925)

161. Mitre, Adolfo. Literatura de guerra. Contra una actitud. (En: Argentina libre, Buenos Aires, 24 oct. 1940)

162. ---, Oliverio Girondo ¡Viva la libertad! (En: Argentina libre, Buenos Aires, 1940)

163. Molina, Enrique. Oliverio Girondo en la noche de los presagios. (En: Amaru, Lima, no 2, abr. 1967, p. 76)

164. --, Palabras. . . (En: Boletîn de la Sociedad Argentina de Escritores, SADE, Buenos Aires,1961-1963, pp. 129-130)

Forma parte del homenaje a Oliverio Girondo realizado en la SADE, el 30 de noviembre de 1962, celebrando sus Veinte poemas para ser leidos en el tranvia.

165. Montenegro, Adelmo R. Los libros: "Persuasión de los dias", de Oliverio Girondo. (En: Córdoba, Córdoba, 5 oct. 1942)

166. Nahuelpan. Diez minutos con Oliverio Girondo, fuerte mentalidad agridulce. (En: Columbia, Buenos Aires, a. 1 no 3, 2 jun. 1925)

167. Neruda, Pablo. Oliverio. (En: Sur, Buenos Aires, no 313, jul.ag. 1968, pp. 1-4)

Poesǐa sobre Oliverio Girondo, fechada en Isla Negra, agosto de 1968.

168. Noé, Julio. Algunos libros de 1923. (En: Nosotros, Buenos Aires, a. 17, v. 45, no 175, dic. 1923, pp. 471-479)

Veinte poemas para ser leidos en el tranvia, por $\mathrm{O}$. Girondo.

169. ---, Teatro nacional. (En: Nosotros, Buenos Aires, a. 9, v. 20, no 80, dic. 1915, pp. 320-322) 
La madrastra, drama en tres actos, de Oliverio Girondo y René Zapata Quesada.

170. Orozco, Olga. En la masmédula. (En: Macedonio, Buenos Aires, a. 1, no 3 , invierno, 1969 , pp. 69-73)

171. --, Poesia y verdad. (En: Claudia, Buenos Aires, a. 11, no 120 , mayo 1967, pp. 42-43)

172. Pagés Larraya, Antonio. Perfil de las letras argentinas. (En: Cuadernos hispanoamericanos, Madrid, no 178, oct. 1964, pp. 77-106)

173. Paseyro, Ricardo. Presentación y elogio de Oliverio Girondo. (En: Indice de artes y letras, Madrid, a. 10, no 95-96, 1956-1957, p. 27)

174. Payró, Julio E. "Colección infantil", de Editorial Sudamericana. (En: Sur, Buenos Aires, no 63, dic. 1939, pp. 71-74)

El autor, que firma J.E.P., se refiere a los cuatro primeros libros de esta colección, dirigida por Oliverio Girondo.

175. Pellegrini, Aldo. Nuevos poemas de Oliverio Girondo. (En: Letra y línea, Buenos Aires, a. 1, no 2, nov. 1953, p. 2)

Se refiere a varios poemas de O. Girondo incluidos en su En la masmédula.

176. Pereda Valdés, Ildefonso. Veinte poemas para ser leidos en el tranvia. (En: La Cruz del Sur, Montevideo, a. 1, no 5, 15 jul. 1924)

177. Petit de Murat, Ulises. "Campo nuestro", de Oliverio Girondo, otro libro de la generación de "Martin Fierro". (En: Crìtica, Buenos Aires, 13 dic. 1946)

178. Pineta, Alberto. La promesa de la nueva generación literaria. (En: Sĭntesis, Buenos Aires, a. 3, no 29, oct. 1929, pp. 207-218)

179. Pinto, Juan. La generación literaria del 22; Oliverio Girondo, poeta surrealista. (En: Clarǐn, Buenos Aires, 27 jul. 1947)

180. Piñero, Sergio. "Calcomanias", por Oliverio Girondo. (En: La Razón, Buenos Aires, 21 jun. 1925)

181. Prieto, Adolfo. El martinfierrismo. (En: Revista de literatura argentina e iberoamericana, Mendoza, Universidad Nacional de Cuyo, Facultad de Filosofía y Letras, Instituto de Lenguas y Literaturas Modernas, a. 1, no 1, 1959, pp. 9-31)

182. Reyes, Salvador. Oliverio Girondo. (En: Los Tiempos, Santiago de Chile, 23 jul. 1924)

183. Rodríguez Fernández, Mario. Oliverio Girondo, "En la masmédula". (En: Anales de la Universidad de Chile, Santiago de 
Chile, a. 114, no 102, 1956, pp. 125-128)

184. Rojas Paz, Pablo. Oliverio Girondo. (En: El Tiempo, Azul, prov. Buenos Aires, 31 ag. 1939)

185. Rosales, César. Oliverio Girondo: "Campo nuestro". (En: Sur, Buenos Aires, no 151, mayo 1947, pp. 103-108)

186. Rossler, Osvaldo. Oliverio Girondo o los peligros del lenguaje. (En: Clarín, Buenos Aires, 9 marzo 1967)

187. Sola, Graciela de. Oliverio Girondo y su libro "En la masmédula". (En: Señales, Buenos Aires, no 148, en.-marzo 1965, pp. 1-4)

188. Sola González, Alfonso. Oliverio Girondo, iniciador de la vanguardia poética argentina. (En: Cuadernos hispanoamericanos, Madrid, v. 55, no 163-164, jul.-ag. 1963, pp. 83-101)

189. Soto, Luis Emilio. Persuasión de los dias. (En: Argentina libre, Buenos Aires, 5 nov. 1942)

190. Soto, Luis Emilio. Texto de Luis Emilio Soto: Para un perfil de Oliverio Girondo. (En: Revista Iberoamericana, Pittsburgh, University of Pittsburgh, v. 41, no 91, abr.jun. 1975, pp. 289-292)

Con una introducción de Juan Loveluck, titulada: La conversación infinita de Luis Emilio Soto. Un texto sobre Oliverio Girondo, pp. 287-288.

191. Suárez Calimano, Eduardo. Calcomanias. (En: Sagitario, La Plata, a. 1, no 2, jul.-ag. 1925, pp. 232-233)

192. Supervielle, Jules. Oliverio Girondo. (En: La Revue de l'Amérique Latine, Paris, marzo 1924)

193. Thénon, Susana. Oliverio Girondo; una historia del fervor. (En: Sur, Buenos Aires, no 315, nov.-dic. 1968, pp. 82-87)

A propósito de la publicación de las Obras completas de Oliverio Girondo.

194. Torre, Guillermo de. Oliverio Girondo. (En: Alfa, Montevideo, abr. 1925)

195. --, ---. (En: Proa, Buenos Aires, a. 2, no 12, jul. 1925, pp. 18-27)

196. Tudela, Mariano. El porteño Oliverio Girondo. (En: Arriba, Madrid, 29 en. 1967)

197. Vasco, Juan Antonio. Oliverio Girondo o la pervivencia del deseo. (En: Testigo, Buenos Aires, no 2, abr.jun. 1966, pp. 40-44) 
Caricatura de Oliverio Girondo en la época de Martin Fierro, por Francisco A. Palomar.

198. Victoria, Marcos. Un experimento de Oliverio Girondo. (En: Clarïn, Buenos Aires, 17 en. 1958)

Sobre En la masmédula.

199. ---, El humorismo en la literatura argentina actual. (En: Cuadernos americanos, México, a. 2, v. 11, no 5, set.-oct. 1943, pp. 206-221)

Sobre O. Girondo, pp. 217-218.

200. Villaurrutia, Xavier. Oliverio Girondo. (En: El Universal, México, 5 oct. 1924)

201. Zỉa, Lizardo. El mundo poético de Oliverio Girondo. (En: Cabildo, Buenos Aires, a. 1, no 53, 15 nov. 1942)

202. Zolezzi, Emilio. Oliverio Girondo. (En: Macedonio, Buenos Aires, a. 1, no 3 , invierno, 1969 , pp. 74-78)

Trabajos sin firmar (orden cronológico)

203. Oliverio Girondo: "Veinte poemas para ser leidos en el tranvia". (En: La Nación, Buenos Aires, 23 dic. 1923, p. 9)

Tomado de la revista La Pluma, jun. 1923.

204. Oliverio Girondo. (En: Martǐn Fierro, Buenos Aires, 2. ép., a. 1, no 2, 20 marzo, 1924)

Busto de Oliverio Girondo, por Mateo Hernández.

205. Oliverio Girondo en misión intelectual. (En: Martín Fierro, Buenos Aires, 2. ép., a. 1, no 7, 25 jul. 1924)

206. Oliverio Girondo en México. (En: Martỉn Fierro, Buenos Aires, 2. ép., a. 2, no 14-15, 24 en. 1925)

207. Libros que se anuncian en 1925. (En: Martìn Fierro, Buenos Aires, 2. ép., a. 2, no 14-15, 24 en. 1925)

Crítica, ensayos, información: Membretes (Condensaciones críticas, de arte y literatura).

Poesía: España, paisaje alucinado. Ed. española.- Veinte poemas para ser leidos en el tranvia. 2. ed. popular.

208. Notas de "Martín Fierro". (En: Martín Fierro, Buenos Aires, 2. ép., a. 2, no 16, 5 mayo 1925)

"El sábado 2 de mayo se efectuó la comida mensual de los componentes de Proa y Martin Fierro, siéndole dedicada a 
Oliverio Girondo de regreso de la jira! americana-europea donde llevó la representación de las revistas jóvenes de Buenos Aires, La Plata y Montevideo."

Se incluye también Oliverio Girondo, poesía de Leopoldo Marechal.

209. "Calcomanias", por Oliverio Girondo. (En: La Nación, Buenos Aires, 1 jul. 1925)

210. Despedida de Girondo. (En: Martïn Fierro, Buenos Aires, 2. ép., a. 3, no 36, 12 dic. 1926)

Con motivo de su viaje a Europa

211. Interlunio. (En: La Nación, Buenos Aires, 14 ag. 1938)

212. "Persuasión de los dias", por Oliverio Girondo. (En: El Litoral, Santa Fe, 22 oct. 1942)

213. Oliverio Girondo: "Persuasión de los dias". (En: Saber vivir, Buenos Aires, nov. 1942)

214. "Campo nuestro", por Oliverio Girondo. (En: La Nación, Buenos Aires, 1 dic. 1946)

215. En la masmédula. (En: La Razón, Buenos Aires, 15 mayo 1966)

216. En la masmédula. (En: La Razón, Buenos Aires, 17 en. 1967)

217. Oliverio Girondo. Falleció en esta Capital. (En: La Nación, Buenos Aires, 25 en. 1967)

218. Una enorme gratitud por la vida. (En: Confirmado, Buenos Aires, a. 3, no 85, 2 feb. 1967)

Con una caricatura de Toño Zalazar, de 1940.

ARTICULOS Y MENCIONES SOBRE EL AUTOR EN HISTORIAS DE LA LITERATURA, ENCICLOPEDIAS Y DICCIONARIOS

219. Anderson Imbert, Enrique. Historia de la literatura hispanoamericana. 4. ed. México-Buenos Aires, Fondo de Cultura Económica, 1964. 2v. (Breviarios, 89 y 156) v. 2: pp. 14, 15, 20,23 y 74 .

220. Ara, Guillermo. Florida y la vanguardia. (En: Historia de la literatura argentina. Buenos Aires, Centro Editor de América Latina, c. 1968. v. 2, pp. 937-960)

221. Fernández Moreno, César. La poesia argentina de vanguardia. (En: Arrieta, Rafael Alberto, dir. Historia de la literatura argentina. Buenos Aires, Peuser, 1958-60.v. 4, pp. 605-669) 
222. Foppa, Tito Livio. Diccionario teatral del Rio de la Plata. Buenos Aires, Argentores, Ediciones del Carro de Tespis, 1961. 1046 p.

En Zapata Quesada, René, p. 699, figura O. Girondo como colaborador de La madrastra (1915) representada en el Teatro Apolo y de Almas a la moda.

223. Historia de la literatura argentina. Buenos Aires, Centro Editor de América Latina, c. 1967-68. 3 v. ilus.

Sobre Oliverio Girondo véase v. 1,2 y 3.

224. Mastronardi, Carlos. El movimiento de "Martin Fierro". (En: Historia de la literatura argentina. Buenos Aires, Centro Editor de América Latina, c. 1968. v. 2, pp. 913-936)

225. Prieto, Adolfo. Oliverio Girondo. (En su: Diccionario básico de literatura argentina. Buenos Aires, Centro Editor de América Latina, c. 1968. Capítulo; Biblioteca argentina fundamental, 59. pp. 70-71)

226. Salvador, Nélida. Oliverio Girondo. (En: Orgambide, Pedro Yahni, Roberto, dir. Enciclopedia de la literatura argentina. Buenos Aires, Sudamericana, c. 1970.pp. 271-276)

La autora firma: N.S.

En su, Trayectoria poética de Oliverio Girondo (Comentario, Buenos Aires, a. 16, no 69, nov.-dic. 1969, pp. 81-82), se anticipan algunos aspectos de este trabajo.

227. Santillán, Diego Abad de. Oliverio Girondo. (En su: Gran enciclopedia argentina. Buenos Aires, Ediar, 1956-63. v. 3, p. 543)

228. Soto, Luis Emilio. El cuento. (En: Arrieta, Rafael Alberto, dir. Historia de la literatura argentina. Buenos Aires, Peuser, 1958-60. v. 4, pp. 285-450)

Interesa especialmente p. 404.

INDICE ALFABETICO DE AUTORES*

A.G., 104

Abril, Xavier, 1905. 105

---, selec. 47

Aguilera, Ricardo F. 106

Aguirre, Raúl Gustavo, 1927. 107

Aita, Antonio, 1891-1966. 108 
Alberti, Rafael, 1902. 109

Alomar, Gabriel, 1873-1941. 110

Alonso, Amado, 1896-1952, dir. 11

Anderson Imbert, Enrique, 1910. 219

Antologia de la poesia moderna hispanoamericana, tĩt. 59

Ara, Guillermo, 1917. 78, 220

-.., selec. 46, 57

Argentina. Ministerio de Educación y Justicia.

Dirección General de Cultura, ed. 36, 42, 53, 60, 80, 88, 91, 98

---.. --.. Subsecretaría de Cultura, ed. 67, 70-73

---. Ministerio de Relaciones Exteriores y Culto. Dirección General de Relaciones Culturales, ed. 49

--. Secretarìa de Estado de Cultura y Educación, ed. 99

Arrieta, Rafael Alberto. 1888-1968, dir. 221, 228

Aznar, Luis, 1902. 111

Baciu, Stefan, selec. 40

Barletta, Leónidas, 1902-1975. 112

Barrantes Castro, Pedro. 113

Bartholomew, Roy, selec. 38

Bayley, Edgar, 1919. 114

Becco, Horacio Jorge, 1924. 1, 115

---, comp. 58

Bernárdez, Francisco Luis, 1900. 116

Binolo, Miguel, impr. 12

Bioy Casares, Adolfo, 1914, selec. 55

Bolaños, Federico. 117

Bonomi, José, ilus. 7

Borges, Jorge Luis, 1899. 118

---, selec. 55

Borzone, Alberto J., ilus. 35

Braccialarghe, Comunardo véase Testena, Folco, seud.

Bullrich, Eduardo J., dir. 66

Bullrich, Eduardo J. 10

Caillet-Bois, Julio César, 1910, selec. 64

"Calcomanias", por Oliverio Girondo, titt. 209

"Campo nuestro", por Oliverio Girondo, tît. 214

Capdevila, Arturo, 1889-1967. 119

Cárdenas de Monner Sans, María Inés. 120

Carilla, Emilio, 1914. 79

Casasbellas, Ramiro de. 121 
Cassou, Jean, 1897. 122

Colección:

Antologias. 36, 53, 91

Biblioteca Cultura e historia. 51,82

Biblioteca de estudios literarios. 76

Biblioteca del Sesquicentenario. 42, 53, 60, 80, 88

Biblioteca fundamental del hombre moderno. 61

Biblioteca románica hispánica. Antologǐa hispánica. 58

Breviarios. 219

Capìtulo; biblioteca argentina fundamental. 57, 225

Cuadernos del Instituto de Letras, 77

Cuadernos humanitas, 79

Cuadernos Julio Herrera y Reissig. 59

Estudios de literatura. 78

Estudios latinoamericanos. 75

Infantil. 174

El Libro de bolsillo. 56

Mar abierto. 45

Movimientos literarios. 42, 60, 80, 88, 99

Palabra en el tiempo. 74

El Pensamiento argentino. 41

Piragua. 87

Los Poetas. 68-69

Poetas de ayer y de hoy. 6

Poetas de España y América. 5, 11

Las Revistas. 96

Serie del encuentro. 15

Serie del Siglo y medio. 64

Tierra firme. 84

21. 48

El Viento. 47

Colegio de México. México, ed. 81

Colombo, Francisco A., ed. 12

Cometta Manzoni, Aída. 123

Córdova Iturburu, Cayetano, 1902-1977. 10, 42, 80, 124-125

Corro, Gaspar Pỉo del, 1923. 75

Coyné, André. 145

Crespo, Rafael A. 65

Cuadrado, Arturo, dir. 74

Davis, Milton Ben, tr. 72

Delio, H.B. 126 
De Nóbile, Beatriz. 76

Despedida a Girondo, tít. 210

Diego, Celia de. 127

Dǐez Canedo, Enrique, 1879-1944. 81, 128

Donoso, Armando, 1887-1946. 129

Editorial universitaria de Buenos Aires véase Eudeba

Una enorme gratitud por la vida, tìt. 218

En la masmédula, tìt. 215, 216

Erro, Carlos Alberto, 1903-1968. 130

Eudeba, ed. 64

F.B. 131

Fernán dez Castro, Alberto. 132

Fernández Moreno, César, 1919. 51, 82, 133, 221

----, comp. 58

Ferreiro, Alfredo Mario, 1899. 134

Figari, Pedro, 1861-1938.

Fitts, Dudley, ed. 72

Fondo Nacional de las Artes. Buenos Aires, ed. 35

Foppa, Tito Livio, 1884. 222

G.C. 135

Gahisto, Manoel, tr. 31-32, 70

Galtier, Lysandro Z.D., 1902, selec. 67, 70-73

Ganduglia, Santiago. 136

García, Germán. 83

Ghiano, Juan Carlos, 1920. 84

Giménez Caballero, Ernesto, 1899. 137

Gimferrer, Pedro. 138

Girondo, Oliverio, 1891-1967. 1-64, 70-74

-.-, dir. 66, 174

-.-, ilus. 13-14

---, pról. 65

-., tr. 67-69

Girondo, Oliverio, 1891-1967. 75-228

Giusti, Roberto F., 1887. 139

Gómez Carrillo, Enrique, 1873-1927. 140

Gómez de la Serna, Ramón, 1888-1963. 85-87, 141-142

Gómez de la Serna, Ramón, 1888-1963. 20, 96, 98, 137

González, Evaristo véase Méndez, Evar, seud.

González Carbalho, José, 1901-1958, selec. 52 
González Climent, Anselmo, selec. 48

González Contreras, Gilberto. 143

González Lanuza, Eduardo, 1900. 60, 88, 144

Hernández, José, 1834-1886. 21

Hernández, Mateo. 204

Historia de la literatura argentina, tít. 220, 223-224

Ibarra, Néstor. 89

Interlunio, tït. 211

Isaacson, José, 1922, selec. 37

J.E.P.

véase Payró, Julio $\mathrm{E}$.

J.H.B. 146

Jarnés, Benjamïn, 1888-1950. 147

Jiménez, José Olivio, selec. 56

Lange, Norah, 1906-1972. 90

Latorre, Carlos. 148-149

Ledesma, Roberto, 1901-1966. 91

Libros que se anuncian para 1925, tít. 207

Loprete, Carlos Alberto. 150

Loveluck, Juan, introd. 190

M.C. 151

Madariaga, Francisco. 152

Maldonado Bayley, Edgar véase Bayley, Edgar

Marechal, Leopoldo, 1900-1970. 153, 208

Martín Fierro, Buenos Aires, publ. per. 10, 18, 21, 93, 96, 120, 123, $125,177,197,208$

Martǐnez Cuitiño, Vicente, 1887-1964. 154

Martini Real, Juan Carlos, comp. 50

Maseras, Alfonso, 1884-1939. 155

Mastronardi, Carlos, 1901. 224

Maturo, Graciela

véase además Sola, Graciela de

Maturo, Graciela, dir. 75

Mazzanti, Carlos, dir. 74

Mazzei, Angel, 1920. 92

Méndez, Evar, 1888-1955. 156-158

Méndez, Evar, 1888-1955. 10, 14, 21

Miomandre, Francis de, 1880. 159

Mister Pin. 160 
Mitre, Adolfo, 1910. 161-162

Molina, Enrique, 1910. 1, 163-164

-.-, ilus. 6, 12

,--- tr. 67-69

Montenegro, Adelmo R. 165

Murray, Luis Alberto, 1923, selec. 53

Museo Nacional de Bellas Artes. Buenos Aires 65

N.S.

véase Salvador, Nélida

Nahuelpan. 166

Neruda, Pablo, 1904-1973. 167

Noé, Julio, 1893. 168-169

---, selec. 63

Notas de "Martin Fierro", tit. 208

Ocampo, Silvina, selec. 55

Oliverio Girondo, tìt. 204

Oliverio Girondo en México, tĭt. 206

Oliverio Girondo en misión intelectual, tìt. 205

Oliverio Girondo. Falleció en esta Capital, tǐt. 217

Oliverio Girondo: "Persuasión de los dias", tít. 213

Oliverio Girondo: "Veinte poemas para ser leidos en el tranvia", tít. 203

Onís, Federico de, 1885 , selec. 62

Orgambide, Pedro, 1929, dir. 226

Orozco, Olga. 170-171

Pagés Larraya, Antonio, 1918. 172

Palomar, Francisco, A., ilus. 197

Paseyro, Ricardo. 173

Payró, Julio E. 174

Pellegrini, Aldo, 1903. 36, 175

Pellegrini, Aldo, 1903. 127

Pereda Valdés, Ildefonso, 1899. 176

"Persuasión de los dias", por Oliverio Girondo, tít. 212

Petit de Murat, Ulises, 1907. 177

Pineta, Alberto. 178

Pinto, Juan, 1902. 93-95, 179

Pintura moderna, tít. 65

Piñero, Sergio. m. 1940. 180

La Poesia del siglo veinte en América y España, tĭt. 39

Poesia lírica del siglo XX: España e Hispanoamérica, tĭt. 61

Prebisch, Alberto. 10 
Prieto, Adolfo, 1928. 96, 181

-.-, selec. 41

Proa, Buenos Aires, publ. per. 208

Reyes, Salvador. 182

Reyes Basualto, Neftali Ricardo véase Neruda, Pablo, seud.

Rimbaud, Jean Arthur, 1854-1891. 67-69

Riosca, A., ilus. 2

Rodrĭguez Fernández, Mario. 183

Rojas Paz, Pablo, 1895-1956. 184

Roncal, Pièrre Charles, tr. 29-30, 71

Rosales, César, 1915. 185

---, selec. 49

Rossi de Fiori, Iride, selec. 64

Rossler, Osvaldo, 1926. 186

SADE

véase Sociedad Argentina de Escritores. Buenos Aires

Salas, Horacio, 1938, selec. 45

Salvador, Nélida. 97, 226

Santillán, Diego Abad de, 1897. 227

Scrimaglio, Marta. 77

Seoane, Luis, 1910 , ilus. 74

Sociedad Argentina de Escritores. Buenos Aires. 10

---, ed. 109, 124, 130, 164

Sofovich, Luisa. 98

Sola, Graciela de véase además Maturo, Graciela

Sola, Graciela de. 99, 187

Sola González, Alfonso, 1915. 188

Sorrentino, Fernando, selec. 43-44

Soto, Luis Emilio, 1902-1970. 189, 190, 228

Spilimbergo, Lino Eneas, 1896-1964, ilus. 8

Suárez Calimano, Eudardo, m. 1949. 191

Sucre, Guillermo, 1933. 100

Supervielle, Jules, 1884-1960. 66, 192

Teatro Apolo. Buenos Aires. 222

Testena, Folco, 1875-1951, selec. 73

---, tr. 73

Thénon, Susana. 193

Tiempo, César, 1906, selec. 23, 54

Torre, Guillermo de, 1900-1971. 101, 194-195 
,-- dir. 5,11

-.--, pról. 49

Tudela, Mariano. 196

Universidad Central de Venezuela. Dirección de Cultura. Caracas, ed. 148

Universidad de Buenos Aires. Facultad de Filosofía y Letras. Instituto de Literatura Argentina "Ricardo Rojas", ed. 97

Universidad de Chile. Santiago de Chile, ed. 183

Universidad Nacional de Córdoba, ed. 41

Universidad Nacional de Cuyo. Facultad de Filosofía y Letras.

Instituto de Lenguas y Literaturas Modernas. Mendoza, ed. 181

Universidad Nacional de Tucumán. Facultad de Filosofía y Letras, ed. 79

Universidad Nacional del Litoral. Santa Fe, ed. 120

Universidad Nacional del Litoral. Facultad de Filosofía y Letras.

Instituto de Letras. Santa Fe, ed. 77

Universidad Nacional del Nordeste. Resistencia, ed. 79

Universidad Nacional del Sur. Extensión Cultural. Bahǐa Blanca, ed. 47

University of Pittsburgh, ed. 190

Urquia, Carlos Enrique, selec. 37

Vasco, Juan Antonio. 197

Victoria, Marcos, 1902-1975. 102-103, 198-199

Vignale, Pedro Juan, 1903-1974, selec. 23, 54

Villaurrutia, Xavier. 200

Yahni, Roberto, dir. 226

Zalazar, Toño, ilus. 218

Zapata Quesada, René, 1891-1952, colab. 169, 222

Zeitlin, Israel véase Tiempo, César

Zìa, Lizardo, 1900. 201

Zolezzi, Emilio. 202

* Incluye autores, colaboradores, editores literarios, directores, prologuistas, seleccionadores, compiladores, traductores e ilustradores, asi como organismos nacionales, universidades e instituciones nacionales y extranjeras, títulos de colecciones, diarios y revistas. Para el caso especial de los escritores citados en los asientos bibliográficos, se mencionan separadamente y los nombres van en versalita. Los números en bastardilla indican fechas y los siguientes remiten a los asientos respectivos. 


\section{BIBLIOGRAFÍA}

ABREVTATURAS

$\begin{array}{ll}\text { a. } & \text { año } \\ \text { abr. } & \text { abril } \\ \text { ag. } & \text { agosto } \\ \text { as. } & \text { asiento,-s } \\ \text { c. } & \text { copyright } \\ \text { cm. } & \text { centïmetro,-scm. } \\ \text { comp. } & \text { compilador, compilación } \\ \text { dic. } & \text { diciembre } \\ \text { dir. } & \text { director,-es; dirigida } \\ \text { ed. } & \text { editor, edición, editado } \\ \text { en. } & \text { enero } \\ \text { ép. } & \text { época } \\ \text { f. } & \text { fases } \\ \text { feb. } & \text { febrero } \\ \text { fév. } & \text { février } \\ \text { ilus. } & \text { ilustración,-es; ilustrador } \\ \text { impr. } & \text { imprenta, impresor, impreso } \\ \text { introd. } & \text { introducción } \\ \text { jul. } & \text { julio } \\ \text { jun. } & \text { junio }\end{array}$

lám.

lámina,-s

m.

$\mathrm{n}^{\circ}$.

murió, muerto

nov.

número,-s

oct.

noviembre, novembre

orig.

octubre, octobre

p.

original

pról.

prov.

página,-s

publ. per. publicación periódica

r.p.m.

retr.

s.e.

selec.

set.

supl.

prólogo, prologuista

provincia

revoluciones por minuto

retrato

sin editor

t.

tìt.

tr.

selección, selector

setiembre

suplemento

tomo,-s

título

traductor, traducción, traducido

v.

volumen,-es 
